It is found that, in these intervals :

$$
\begin{aligned}
& F_{i}\left(z_{0}, z, t\right)= \\
& \sum_{r=0}^{\infty} e^{-\lambda\left\{\left[\frac{1}{2}(r+1)\right] \alpha_{i}+\left[\frac{1}{2} r\right] a j-z\right\}} \\
& \left(\lambda\left\{\left[\frac{1}{2}(r+1)\right] \alpha_{i}+\left[\frac{1}{2} r\right] \alpha_{j}-z\right\}\right)^{r} / r ! \times \\
& \left\{\frac{\delta_{r}}{2},\left[\frac{r}{2}, F_{i}\left(z_{0}, 0, z+t-\left[\frac{1}{2}(r+1)\right] \alpha_{i}-\left[\frac{1}{2} r\right] \alpha_{j}\right)+\right.\right. \\
& \left.\left(1-\frac{\delta_{r}}{2},\left[\frac{r}{2}\right]\right) F_{j}\left(z_{0}, 0, z+t-\left[\frac{1}{2}(r+1)\right] \alpha_{i}-\left[\frac{1}{2} r\right] \alpha_{j}\right)\right\}
\end{aligned}
$$

where $i, j=1,2$, but $i \neq j$. From these, the distribution function $F\left(z_{0}, z, t\right)$ of $(3)$ can be obtained; in the case $\alpha_{1}=\alpha_{2}$, this naturally reduces to the form previously given by Gani and Prabhu ${ }^{6}$.

The method outlined is equally applicable to the case of $p>2$ ordered inputs $\alpha_{1}, \ldots, \alpha_{p}$. There is a corresponding increase in the complexity of the equations for the associated probabilities of first emptiness starting with an input $\alpha_{1}$, as well as for the distribution function of the process.

Stanford University,

J. GANI

California, and

University of Western Australia, Perth.

${ }^{1}$ Takács, L., Acta Math. Acad. Sci. Hungaricae, 6, 101 (1955). "Gani, J. "First Emptiness of Two Dams in Parallel" (to be pub-

${ }^{3}$ Kendall, D. G., J. Roy. Statist. Soc., B, 19, 207 (1957).

- Gani, J., Math. Ann., 136, 454 (1958).

"Gani, J., and Pyke, R., "Inequalities for First Emptiness Probabilities of a Dam with Ordered Inputs" (to be published). - Gani, J., and Prabhu, N. U., J. Math. Mech., 8, 653 (1959).

\section{Plant Population and Crop Yield}

UnDER the above title, Holliday ${ }^{1}$ has recently published an equation which describes in a number of cases the relationship between yield and density in crop plants. In its simplest form this relationship is :

$$
1 / w=A \rho+B
$$

where $w$ is the yield per plant, $\rho$ is the number of plants per unit area and $A$ and $B$ are constants. This equation was used (with these symbols) by Shinozaki and Kira ${ }^{2}$ in 1956. An equivalent relationship connecting the total yield per unit area, $y=\rho w$, with the spacing per plant, $s=1 / \rho$, namely:

$$
y=\frac{1}{A+B s}
$$

was proposed independently be De Wit and Ennik in $1958^{3}$. These authors concede that the formula may not apply at high plant densities, and Holliday has directed attention to its limitations at very low plant densities, and accordingly has proposed a modification of equation (1). Appropriate modifications for both extremes are considered by Shinozaki and Kira ${ }^{2}$. Holliday further points out that even his modified equation cannot describe adequately total yield/plant density relationships where the form of the curve is parabolic, that is, where a certain plant population gives higher yields than either lower or higher populations. This type of curve, it is claimed, is typical of situations where yield is a function of reproductive growth, whereas curves that reach a stable maximum with increasing plant density are typical of yield of vegetative growth, and are defined by equations similar to or derived from equation (1).

There appear to be exceptions to this generalization; thus the data of Wiggins ${ }^{4}$ for the yield of threshed beans from soybean are adequately described by equation (1), whereas our own observations (unpublished) show that the yields of onions and lettuce frequently are not.

The Japanese contribution to knowledge of this subject does not appear to be widely known, and we therefore feel that the references given below ${ }^{5}$ may be useful. Kira and his co-workers have shown how equation (1) can be derived from the logistic equation for plant growth together with certain other plausible assumptions, and have studied how the parameters in the equation change with time.

Hozumi, Kira and Shinozaki ${ }^{6}$ have developed the application of equation (1) to cover the effects of any growth factor in suboptimal supply using the form :

$$
\frac{1}{w}=\frac{A}{f}+B
$$

where $f$ is the amount of a factor supplied. They have developed the generalization for several factors varying simultaneously, using multilinear forms in the inverses of the amounts of the factors. Thus for two factors of amounts $f_{1}$ and $f_{2}$, they put:

$$
\frac{1}{w}=\frac{A_{1}}{f_{1}}+\frac{A_{2}}{f_{2}}+\frac{A_{12}}{f_{1} f_{2}}+B
$$

and show that this model successfully describes the joint effect of light intensity and nutrient concentration on the growth of Lemna minor ${ }^{7}$, and the joint effect of light intensity and density on the growth of Hibiscus moscheutos ${ }^{6}$. Although Kira and his co-workers have apparently successfully used equation (1) and their generalization of it for a number of species, we find that the relation :

$$
\frac{1}{w^{\theta}}=A \rho^{\theta}+B
$$

where $\theta$ is some positive quantity usually less than unity, frequently gives a strikingly better fit to data from spacing experiments. Equation (3) can be derived by analogous arguments to those of Kira et al., the logistic equation being replaced by a generalization described by Richards ${ }^{8}$. By allowing the power of $\rho$ in (3) to exceed that of $w$, a model can be obtained in which the yield per unit area shows a maximum at a certain finite density. A detailed account of this work will be published elsewhere.

\section{J. K. A. Bleasdale}

$$
\text { J. A. NELDER }
$$

National Vegetable Research Station, Wellesbourne, Warwick.

' Holliday, R., Nature, 188, 22 (1960).

'Shinozaki, K., and Kira, T., J. Inst. Polytech., Osaka, D, 7, 35 (1956).

${ }^{3}$ Wit, C. T. de, and Ennik, G. C., Jaarb. Inst. biol. scheik. Onderz. LandbGewass. for 1958, 59 (i959).

'Wiggins, R. G., J. Amer. Soc. Agron., 31, 314 (1939).

${ }^{5}$ Kira, T., Ogawa, H., and Sakazaki, H., J. Inst. Polytech., Osaka, D, 4,1 (1953). Kira, T., Ogawa, H., and Hozumi, K., ibid., D, 5 1 (1954). Ikusima, I., Shinazaki, K., and Kira, T., ibid., D, 6. 107 (1955). Hozumi, K., Koyama, H., and Kira, T., ibid., D, 6, 121 (1955). Kira, T., et al. ibid., D, '7, 1 (1956). Hozumi, K., Asahira, T., and Kira, T., ibid., D, 7, 15 (1956). Koyama, H. and Kira, T., ibid., D, ${ }^{2}, 73$ (1956). Yoda, K., Kira, T., and Hozumi, K., ibid., D, 8, 161 (1957).

' Hozumi, K., Kira, T., and Shinozaki, K., Seivo-Seitai, 8, 36 (1958).

"Ikusima, I., and Kira, T., Seivo-Seitai, 8, 50 (1958).

8 Richards, F. J., J. Exp. Bot., 10, 290 (1959). 